"Der Gehalt des „Polysulfins" an Schwefelnatrium ist ein sehr geringer. Wir können nicht annehmen, dass 1/, Proc. Schwefelalkali eine besonders reinigende Wirkung auf die Wäsche ausübt; die günstigen Wirkungen des "Polysulfins" sind nur dem Umstande zuzuschreiben, dass das „Polysul fin" eine feinpulverige Soda*darstellt, welche in richtiger Verdünnung angewandt wird."

Die „Aufklärung“ über den wahren Charakter des "Polysulfins", welche B. Fi scher in dem Gutachten der Prüfungsstation vermisst, ist also der Heidelberger Firma bereits i. J. 1894 im vollsten Masse zu Theil geworden. Wenn nun diese Firma gleichwohl behauptet, dass die reinigenden und bleichenden Wirkungen des "Polysulfins" durch die Eigenart der Polysulfide des Kaliums und Natriums bedingt seien, oder wenn sie im Gegensatze hierzu ferner angibt, die guten Wirkungen des "Polysulfins" seien den - für Waschzwecke ganz wertblosen "Zersetzungsproducten der Polysulfide" zuzuschreiben, so werden derartige Behauptungen, wie aus Vorstehendem zweifellos hervorgeht, wider besseres Wissen gemacht.

Nur beiläufig sei bemerkt, dass das "Polysulfin" nach B. Fischer (1896/97) 7,5 Proc. Natriumcarbonat weniger und 8,2 Proc. Wasser mehr enthält wie das von der Station i. J. 1894 untersuchte Muster. Es

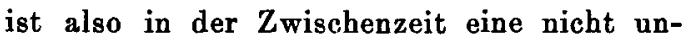
erhebliche Verschlechterung dieser Waare eingetreten.

$\mathrm{Ob}$ es nicht möglich ist, gegen die Heidelberger Firma, welche über die Beschaffenheit und die Herstellungsart des "Polysulfins" nachweislich unrichtige und mit der Analyse der Prüfungsstation im Widerspruch stehende Angaben thatsächlicher Art macht, auf Grund der $\S \S 1$ und 4 des Gesetzes zur Bekämpfung des unlauteren Wettbewerbs vom 27. Mai 1896 erfolgreich vorzugehen, mag an dieser Stelle unerörtert bleiben.

Darmstadt, im Mai 1898.

$$
\text { Dr. W. Sorne. }
$$

\section{Über eine Verbesserung am Soxhlet'schen Extractionsapparat.}

$$
\text { Von }
$$

\section{Dr. H. Weller in Darmstadt.}

Im Heft 6 S. 160 d. Z. hatte Dr. Louise einen verbesserten Soxhlet'schen Apparat zur Extraction von Fetten beschrieben, welcher sich von dem gewöhnlichen Soxhlet'schen Apparat dadurch unterscheidet, dass in der Mitte des
Heberrohres ein Glashahn angebracht ist, um nach Beendigung der Extraction den Äther nach dem Schliessen des Hahnes abzudestilliren.

Schon i. J. 1890 habe ich mir von der Firma Ehrhardt \& Metzger in Darmstadt eine Anzahl derartiger Apparate mit Hähnen herstellen lassen und zwar ebenfalls zuerst in der Mitte des Heberrohres, später der geringeren Zerbrechlichkeit wegen an dem unteren Theile desselben.

In dem Preisverzeichnisse von Ehrhardt \& Metzger auf S. 78 No. 759 vom Jahre 1890 ist bereits der von mir eingeführte Apparat abgebildet und habe ich damals eine Beschreibung dieses Apparates unterlassen, weil ich der Ansicht bin, derartige kleine Änderungen an Apparaten in wissensehaftlichen Zeitschriften nicht besonders hervorheben zn müssen.

Zum Schlusse will ich noch bemerken, dass ich seit dieser Zeit diese Apparate in unserem Institute führe und mit denselben sehr zufrieden bin.

Darmstadt, im Juni 1898.

\section{Über die Ertheilung von Zusatzpatenten. Von}

\section{Dr. Edg. Odernheimer.}

Durch den interessanten Aufsatz von E. Kloeppel (d. Z. Heft 18) ist darauf hingewiesen worden, mit welcher Schärfe das Patentamt in neuerer Zeit bei Ertheilung von Zusatzpatenten prüf, ob durch diesen Zusatz auch ein wirklich neuer Effect dem Hauptpatente gegenüber erzielt wird.

Schon in früheren Jahren ist dieser Standpunkt vom Patentamte ausdrücklich hervorgehoben worden, indem betont wurde, dass das Zusatzpatent nicht benutzt werden soll, um Versehen, welche bei der Abfassung des Hauptpatentes begangen sind, $\mathbf{z u}$ berichtigen, insbesondere nicht die Gültigkeitsgrenzen des Hauptpatentes nachträglich zu erweitern. (P.-Bl. 1881 S. 29, G. 2, 97.) S. Patentgesetz erläutert von Dr. A. Seligsohn, Bd. I S. 85 .

Auch nach meiner Ansicht lässt sich eine so strenge Auffassung nicht durch den Wortlaut des Paragraphen 7 der Patentgesetzgebung begründen.

\section{Htittenwesen.}

Bessemerbirne. Nach R. M. Daelen (D.R.P. No. 97014 ) muss eine Birne, welche ähnlich einer Bessemerbirne zum Vorfrischen von flüssigem, aus einem Hoch- oder Cupolofen entnommenen Robeisen dienen soll, welches dann behufs Weiterverarbeitung zu Flusseisen in einen Herdofen abgegeben wird, einen sehr grossen inneren Raum besitzen, um ein Überkochen zu verhindern; infolge 